\title{
Administration of Lactobacillus gasseri SBT2055 suppresses macrophage infiltration into adipose tissue in diet-induced obese mice
}

\author{
Ken Ukibe*, Masaya Miyoshi and Yukio Kadooka \\ Milk Science Research Institute, Megmilk Snow Brand Co. Ltd, 1-1-2 Minamidai, Kawagoe, Saitama 350-1165, Japan \\ (Submitted 30 September 2014 - Final revision received 26 May 2015 - Accepted 18 June 2015 - First published online 24 August 2015)
}

\section{Abstract}

Administration of Lactobacillus gasseri SBT2055 (LG2055) has been shown to prevent body weight gain and it also down-regulates the expression of the $\mathrm{Ccl} 2$ gene in adipose tissue in diet-induced obese mice. The CC chemokine ligand 2 has a crucial role in macrophage infiltration into adipose tissue, which is known to exacerbate inflammation. However, it is not yet known how LG2055 affects the invasion of macrophages into adipose tissue. C57BL/6J male mice were fed a normal-fat diet (10\% energy fat), high-fat diet (HFD; $45 \%$ energy fat), or HFD containing LG2055 for 12 weeks. After the feeding period, gene expression and macrophage population in adipose tissue were analysed by real-time PCR and flow cytometry, respectively. Body weight and abdominal fat weight were not altered by feeding LG2055. Flow cytometry analysis revealed that the population of macrophages in adipose tissue was significantly reduced by feeding LG2055 compared with HFD only. Furthermore, the ratio of classically activated inflammatory macrophages (M1 macrophages) to total macrophages was significantly decreased in the LG2055-fed group. The expressions of Ccl2, Ccr2 and Lep were down-regulated and that of Il6, Tnf and Nos 2 tended to be down-regulated in adipose tissue by feeding LG2055. In addition, fasting glucose levels were significantly decreased in the LG2055-fed group. These data suggest that administration of LG2055 might attenuate inflammation, which is caused by the intake of an HFD, through the inhibition of macrophage invasion into adipose tissue.

\section{Keywords: Probiotics: Macrophages: Inflammation: Obesity}

Lactic acid bacteria (LAB) are defined as bacteria that produce lactic acid as their main fermentation product. They have been traditionally used in the manufacture of various types of food products such as pickles, cheese and yogurt and are recognised as safe and beneficial bacteria ${ }^{(1)}$. It has been recognised that some LAB can be used as probiotics, which have valuable effects on human health: the WHO has defined probiotics as 'Live microorganisms that, when administered in adequate amounts, confer a health benefit to the host ${ }^{,(2)}$. A large number of studies have reported that probiotic strains of LAB exhibit beneficial effects such as prevention of diarrhoea, allergic disease and infectious diseases ${ }^{(3,4)}$; several strains of LAB have also been recently reported to have an anti-obesity effect ${ }^{(5-9)}$.

Lactobacillus gasseri SBT2055 (LG2055) is a probiotic bacterium isolated from human faeces. It has been shown that oral administration of LG2055 beneficially changes the composition of human intestinal microflora and the physical characteristics of faeces ${ }^{(10)}$, and that the administration prevents mice from influenza infection ${ }^{(11)}$. The anti-obesity effect of this bacterium has been demonstrated in both rats and humans ${ }^{(12-16)}$. Miyoshi et $a l^{(17)}$ recently confirmed an anti-obesity effect of LG2055 in diet-induced obese mice, and also reported that the expression of the $\mathrm{Ccl} 2$ gene in adipose tissue was down-regulated by the administration of LG2055.

Under the obese state, adipocytes secrete free fatty acids and pro-inflammatory cytokines such as TNF- $\alpha$, IL- 6 and CC chemokine ligand 2 (CCL2, the translational product of the $\mathrm{Ccl} 2$ gene is also known as monocyte chemoattractant protein 1 (MCP-1) $)^{(18)}$. CCL2 leads to infiltration of monocytes expressing CCR2 (CC chemokine receptor 2) into adipose tissue, where the infiltrated monocytes differentiate into classically activated inflammatory macrophages (M1 macrophage). Under the lean state, however, most macrophages in the adipose tissue show an alternatively activated anti-inflammatory macrophage (M2 macrophage) phenotype. Consequently, the ratio of the M1:M2 phenotype is increased with development of obesity $^{(19)}$. M1 macrophages also secrete TNF- $\alpha$, IL-6 and CCL2: TNF- $\alpha$ can further induce the secretion of pro-inflammatory cytokines including CCL2 ${ }^{(18)}$. Inflammation is, thus, exacerbated by this 'feed-forward loop' in obese adipose tissue, and the augmented inflammation leads to the development of obesityassociated insulin resistance and type 2 diabetes ${ }^{(20,21)}$. It was

Abbreviations: CFU, colony-forming units; HFD, high-fat diet; HFLGD, HFD containing LG2055; HOMA-IR, homoeostasis model assessment for insulin resistance; LAB, lactic acid bacteria; LG2055, Lactobacillus gasseri SBT2055; NFD, normal-fat diet; SVF, stromal vascular fraction.

* Corresponding author: K. Ukibe, fax +81 49242 8157, email ken-ukibe@meg-snow.com 
also reported that transgenic over-expression of CCL2 increased macrophage infiltration and inflammation ${ }^{(22)}$, whereas the knock down of CCL2 and CCR2 depressed migration of macrophages into adipose tissue and improved insulin sensitivity $^{(23,24)}$.

Given that LG2055 has previously been shown to downregulate the expression of the $C c l 2$ gene $^{(17)}$, the translational product of which is a crucial factor for macrophage infiltration, it is of interest to examine whether the administration of LG2055 actually suppresses macrophage invasion into adipose tissue, which has not been studied thus far. We investigated the effect of administration of LG2055 on macrophage infiltration and ratio of the M1:M2 phenotype in adipose tissue, as well as systemic insulin sensitivity, using HFD-induced obese mice.

\section{Methods}

\section{LG2055 cells}

LG2055 was cultured in deMan, Rogosa and Sharpe (MRS) broth (BD Bioscience) for $18 \mathrm{~h}$ at $37^{\circ} \mathrm{C}$. LG2055 cells were collected by centrifugation at $3000 \mathrm{~g}$ for $10 \mathrm{~min}$ at $4^{\circ} \mathrm{C}$ and washed three times (twice with saline and once with distilled water). Finally, the cells were suspended in 5 volumes of $10 \%(\mathrm{w} / \mathrm{v})$ lactose solution and lyophilised using a FDU-2200 freeze-dryer (Tokyo Rikakikai Co. Ltd). The colony-forming units (CFU) of dried LG2055 powder, determined using an MRS agar plate, was $1 \times 10^{11} \mathrm{CFU} / \mathrm{g}$.

\section{Diets and animals}

A high-fat diet (HFD) containing $45 \%$ energy fat (D12451) and a low-fat control diet containing $10 \%$ energy fat (D12450H) were purchased from Research Diets Inc.; $1 \%(\mathrm{w} / \mathrm{w})$ of the dried LG2055 powder was mixed with the diet containing $45 \%$ energy of fat to produce the HFD containing LG2055 (HFLGD). The CFU of this diet, determined using an MRS agar plate, was $1 \times 10^{9} \mathrm{CFU} / \mathrm{g}$. The normal-fat diet (NFD) and HFD were prepared using $1 \%(\mathrm{w} / \mathrm{w})$ lactose mixed with the $10 \%$ energy fat diet and $45 \%$ energy fat diet, respectively. No LG2055 powder was mixed into the NFD and HFD.

Six-week-old male C57BL/6J mice (weaned at 3 weeks of age) were purchased from Charles River Japan Inc. Mice were individually housed in an air-conditioned, specific pathogenfree room $\left(21-24^{\circ} \mathrm{C}\right.$, lights on $08.00-20.00$ hours). After 1 week of acclimatisation, mice were divided into three groups ( $n 12$ in each group), each fed the NFD, HFD or HFLGD. During the feeding period, mice were given free access to the experimental diet and water. Dietary amounts initially dispensed and residual diet after consumption were recorded every day, and the amount of food consumed was calculated by subtraction of the amount of residual diet from that initially dispensed. The amount of diet dispensed was daily adjusted by pair-feeding so that there were no differences in dietary intake between the HFD- and HFLGD-fed groups, whereas the amount dispensed for the NFD-fed group was not adjusted. At the start of the experiment, the amount of diet dispensed was $4.0 \mathrm{~g} / \mathrm{d} ; 1$ week later, the amount was adjusted to $3.5 \mathrm{~g} / \mathrm{d}$, because the mean diet intake of both groups did not exceed $3.5 \mathrm{~g}$. From weeks 1 to 6 , the HFLGD group tended to consume more food compared with the HFD group, although there was no significant difference; subsequently, from week 6 to the end of the experiment, the amount of diet dispensed was reduced to $3.0 \mathrm{~g} / \mathrm{d}$ according to the mean diet intake in the HFD group. Body weight of mice was monitored weekly.

At the end of the feeding period, mice were anaesthetised by diethyl ether and killed by cervical dislocation after fasting for $12 \mathrm{~h}$. The visceral adipose tissue samples (mesenteric, perirenal/retroperitoneal and epididymal) were dissected and weighed. All mesenteric fat and part of the epididymal fat were frozen in liquid $\mathrm{N}_{2}$ and stored at $-80^{\circ} \mathrm{C}$ for RNA preparation. The remaining section of epididymal fat was suspended in RPMI1640 medium (Life Technologies) containing 10\% (v/v) fetal bovine serum (FBS), 10 mM-HEPES, 2 mM-L-glutamine, $100 \mathrm{U} / \mathrm{ml}$ penicillin, $100 \mu \mathrm{g} / \mathrm{ml}$ streptomycin and $0.05 \mathrm{~mm}-2-$ mercaptoethanol and stored on ice before preparation of the stromal vascular fraction (SVF).

The protocol was in accordance with the animal experimentation regulations of the Milk Science Research Institute of Megmilk Snow Brand Co. which are based on the guidelines proposed by the Science Council of Japan.

\section{Isolation of stromal vascular fraction and flow cytometry analysis}

Isolation of SVF was performed using the method proposed by Nishimura et ll $^{(25)}$, with slight modification. Epididymal fat was transferred into PBS containing $1 \mu \mathrm{g} / \mathrm{ml}$ heparin and minced into small pieces using a pair of scissors. They were vigorously shaken for $30 \mathrm{~s}$ to remove the circulating blood cells and then centrifuged at $370 \boldsymbol{g}$ for $5 \mathrm{~min}$. Floating pieces of adipose tissue were collected into collagenase solution (RPMI1640 containing 10 mM-HEPES, $1 \mathrm{mg} / \mathrm{ml}$ collagenase type II (Sigma-Aldrich) and $10 \mu \mathrm{g} / \mathrm{ml}$ DNase I (F. Hoffmann-La Roche)) and incubated for $20 \mathrm{~min}$ at $37^{\circ} \mathrm{C}$. Adipose tissues were suspended by pipetting and were filtered using a 70- $\mu \mathrm{m}$ mesh (BD Bioscience). After centrifugation, precipitated cells were washed twice with RPMI640 (containing $10 \%$ (v/v) FBS and 10 mm-HEPES), then re-suspended into erythrocyte lysis buffer (168 mm-NH $4 \mathrm{Cl}, 10 \mathrm{~mm}-\mathrm{KHCO}_{3}$ and 0.08 mm-EDTA) and incubated for 3 min. The cells were washed and suspended in FCM buffer (PBS containing $1 \%(\mathrm{v} / \mathrm{v})$ FBS and $\left.0.05 \%(\mathrm{w} / \mathrm{v}) \mathrm{NaN}_{3}\right)$, and the number of cells was counted using a Celltac haematology analyser (Nihon Kohden).

Isolated cells were incubated with anti-mouse CD16/32 (eBioscience) for $30 \mathrm{~min}$ at $4^{\circ} \mathrm{C}$ to block non-antigen-specific binding via the $\mathrm{FC}$ receptor. Cells were washed with FCM buffer and incubated with anti-mouse FITC-labelled CD11b, APC-labelled CD11c, PE-labelled CD206 (all from BD Bioscience) and Brilliant violet 421-labelled F4/80 (BioLegend) for $1 \mathrm{~h}$ at $4^{\circ} \mathrm{C}$. The cells were then washed and suspended in FCM buffer before flow cytometry analysis (FACS Canto II; BD Bioscience). Isotype control antibodies were used to estimate the non-specific binding of target antibodies to cell surface antigens. The 7AAD (BD Bioscience) was used to exclude dead cells. Cells highly labelled with both anti-CD11b and anti-F4/80 antibodies $\left(\mathrm{F} 4 / 80{ }^{\text {high }} \mathrm{CD} 11 \mathrm{~b}^{\text {high }}\right)$ were regarded 
as macrophages ${ }^{(26)}$. Macrophages labelled with the anti-CD11c antibody but not the anti-CD206 antibody were regarded as M1 macrophages, and those labelled with the anti-CD206 antibody but not the anti-CD11c antibody were regarded as M2 macrophages $^{(19)}$.

\section{Gene expression analysis}

Total RNA were isolated from epididymal fat tissue using an RNeasy lipid tissue kit (Qiagen). The quality of RNA was confirmed using an Agilent 2100 Bioanalyzer (Agilent), and RNA with an RNA integrity number above 7 were regarded as intact RNA. Intact total RNA $(2 \mu \mathrm{g})$ was reverse-transcribed to cDNA using a High-Capacity RNA-to-cDNA kit (Life Technologies). Real-time PCR analysis was performed using a TaqMan Fast Advanced Master Mix and Viia7 system (both from Life Technologies). TaqMan primer and probe sets for CC chemokine ligand 2 (Ccl2; Mm00441242_m1), CC chemokine receptor 2 (Ccr2; Mm00438270_m1), CD68 antigen (Cd68; Mm03047340_m1), IL-6 (Il6; Mm00446190_m1), nitric oxide synthase 2, inducible (Nos2; Mm00440502_m1), TNF (Tnf; Mm00443258_m1) and leptin (Lep; Mm00434759_m1) were purchased from Life Technologies. The expression levels of target genes were normalised with the endogenous control genes $^{(27)}$, $\beta$-actin (Actb; Mm01205647_g1) and glyceraldehyde3-phosphate dehydrogenase (Gapdh; Mm99999915_g1), and the data were analysed according to the $2^{-\Delta \Delta C_{t}}$ method using DataAssist v3.0 software (Life Technologies).

\section{Serological analysis}

Blood was obtained from the inferior vena cava at the end of the feeding period, incubated at room temperature for $30 \mathrm{~min}$ to form blood clots and then centrifuged at $13000 \boldsymbol{g}$ for $10 \mathrm{~min}$ to obtain serum samples. Serum samples collected were stored at $-80^{\circ} \mathrm{C}$ until assay. Serum glucose levels were analysed using the DRI-CHEM 7000V and GLU-P-III slide (both from Fujifilm Corporation). Serum insulin was analysed using a Mouse Insulin ELISA KIT (Shibayagi Co. Ltd). The index of homoeostasis model assessment for insulin resistance (HOMA-IR) was calculated using the following formula: fasting glucose $(\mathrm{mmol} / \mathrm{l}) \times$ fasting insulin $(\mathrm{mU} / \mathrm{l}) / 22 \cdot 5^{(28)}$.

\section{Statistical analysis}

Statistical analysis was performed using StatView software package 5.0 (SAS Institute Inc.) and PASW Statistics 17 (SPSS Inc.). Significant differences among experimental groups were analysed using ANOVA, followed by Dunnett's test. In this study, to investigate the effect of administration of LG2055 in mice fed a HFD, the group of mice fed HFD only was regarded as the control group. A value of $P<0.05$ was considered to be statistically significant. Correlation analysis was assessed by the Pearson's test using StatView software.

\section{Results}

Diet intake, body weight and visceral fat weight

Mice were divided into three groups and fed NFD, HFD or HFLGD for 12 weeks. Morphometric and biochemical data of mice are shown in Table 1. Although the dietary intake of the NFD-fed group was significantly higher than that of the HFDfed group, energy intake was vice versa, which was due to the high energy content of HFD. There were no differences in diet and energy intakes between the HFD and the HFLGD groups. Body weight and adipose tissue weight were significantly increased by the feeding HFD compared with feeding NFD. No significant differences in body and adipose tissue weights were observed between the HFD and HFLGD groups.

Flow cytometric analysis of macrophages in epididymal fat

The gating strategy to identify M1 and M2 macrophages is shown in Fig. 1. The number of SVF cells $/ 1 \mathrm{~g}$ of epididymal fat did not differ between the groups (Fig. 2(a)). The percentage of $\mathrm{F} 4 / 80^{\text {high }} \mathrm{CD} 11 \mathrm{~b}^{\text {high }}$ macrophages in the SVF collected from epididymal fat tissue was significantly increased by feeding HFD compared with feeding NFD (Fig. 2(b)). The feeding of HFLGD suppressed the increase in $\mathrm{F} 4 / 80^{\text {high }} \mathrm{CD} 11 \mathrm{~b}^{\text {high }}$ macrophages compared with feeding HFD. The percentage of

Table 1. Morphometric and biochemical markers of mice

(Mean values and standard deviations)

\begin{tabular}{|c|c|c|c|c|c|c|c|c|}
\hline & \multicolumn{2}{|c|}{ NFD } & \multicolumn{2}{|c|}{ HFD } & \multicolumn{2}{|c|}{ HFLGD } & \multicolumn{2}{|c|}{$P(v . \mathrm{HFD})^{*}$} \\
\hline & Mean & SD & Mean & SD & Mean & SD & NFD & HFLGD \\
\hline Average diet intake $(\mathrm{g} / \mathrm{d})$ & $3 \cdot 41$ & $0 \cdot 12$ & 3.02 & 0.14 & 3.06 & 0.07 & $<0.001$ & 0.607 \\
\hline Average energy intake $(\mathrm{kJ} / \mathrm{d})$ & 54.93 & 1.93 & $59 \cdot 77$ & 2.77 & $60 \cdot 56$ & 1.39 & $<0.001$ & 0.566 \\
\hline Initial body weight $(\mathrm{g})$ & 21.43 & 0.74 & 21.72 & 0.70 & 21.79 & 0.64 & 0.499 & 0.945 \\
\hline Final body weight $(\mathrm{g})$ & 28.96 & $1 \cdot 60$ & $36 \cdot 40$ & $2 \cdot 39$ & $35 \cdot 42$ & $2 \cdot 12$ & $<0.001$ & 0.412 \\
\hline \multicolumn{9}{|c|}{ Adipose tissue weight ( $\%$ of final body weight) } \\
\hline Mesenteric & 0.99 & $0 \cdot 21$ & $1 \cdot 35$ & 0.20 & 1.33 & 0.34 & 0.003 & 0.990 \\
\hline Epididymal & $2 \cdot 79$ & 0.59 & $5 \cdot 27$ & 0.70 & 4.87 & $1 \cdot 12$ & $<0.001$ & 0.407 \\
\hline Perirenal/retroperitoneal & 1.08 & $0 \cdot 26$ & $2 \cdot 30$ & 0.22 & $2 \cdot 15$ & 0.57 & $<0.001$ & 0.548 \\
\hline Total & 4.86 & 1.00 & 8.92 & 0.99 & $8 \cdot 36$ & 1.96 & $<0.001$ & 0.522 \\
\hline
\end{tabular}

NFD, normal-fat diet; HFD, high-fat diet (45\% energy); HFLGD, high-fat diet (45\% energy) containing LG2055.

* $P$ values were calculated using Dunnett's test. 

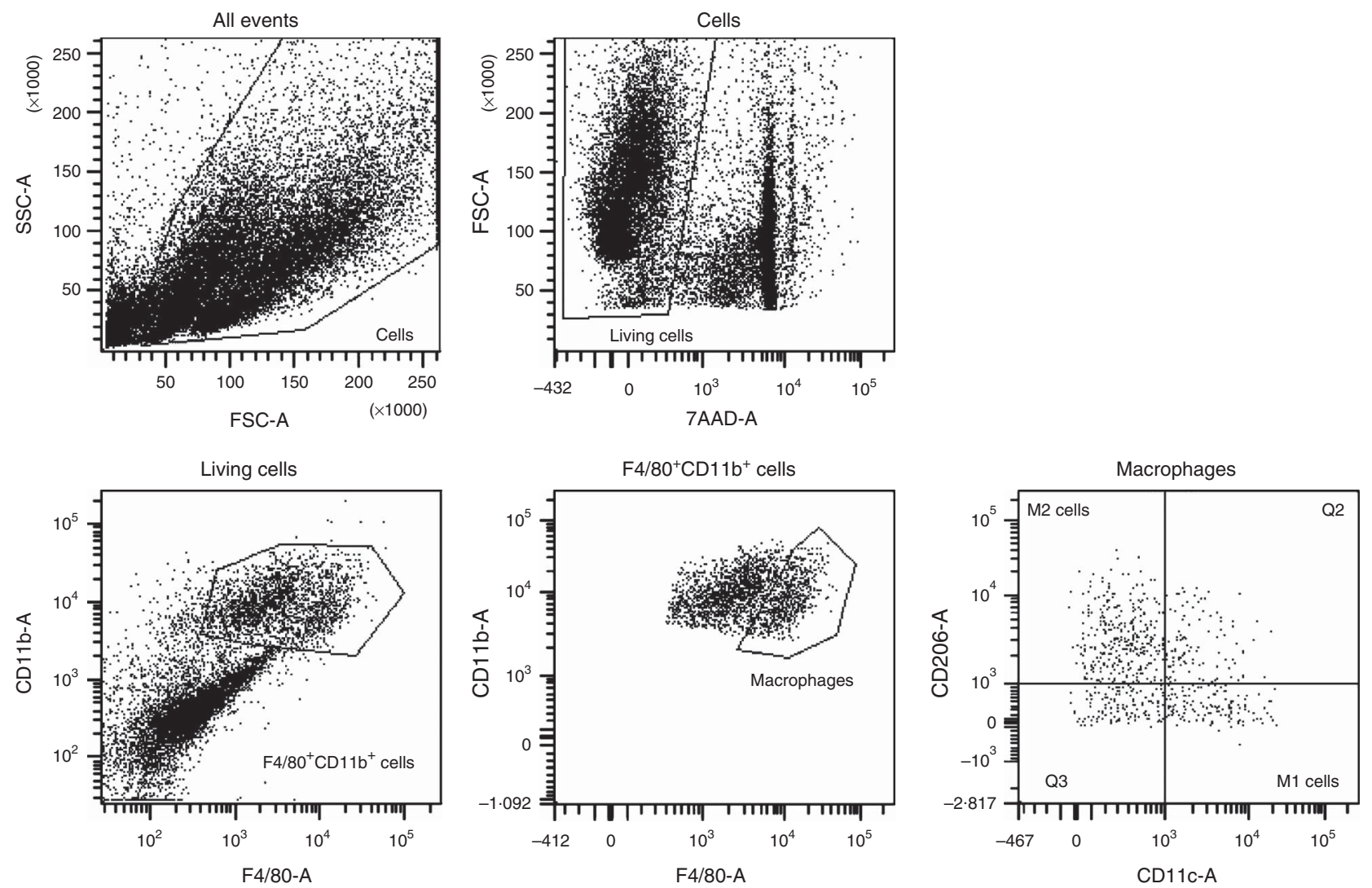

Fig. 1. Gating strategy to identify M1 and M2 macrophages. Stromal vascular fraction cells labelled with anti-F4/80, anti-CD11b, anti-CD11c and anti-CD206 antibodies were analysed using a FACS Canto II flow cytometer. Cell debris and dead cells $\left(7 A A D^{+}\right)$were excluded in the first step. $F 4 / 80^{\text {high }} C D 11 b^{\text {high }}$ cells were identified as macrophages and gated to expand on a new cytogram presenting CD11c and CD206 as axes. CD11c ${ }^{+}$CD206 ${ }^{-}$cells and CD11c ${ }^{-}$CD206 ${ }^{+}$cells were identified as M1 and M2 macrophages, respectively. Isotype controls were used to set the lines separating positive cells from negative cells.

CD11 ${ }^{+} \mathrm{CD} 206^{-}$cells (M1 macrophages) to the $\mathrm{F} 4 / 80^{\text {high }} \mathrm{CD} 11 \mathrm{~b}^{\text {high }}$ macrophages was also significantly increased in mice fed HFD compared with mice fed NFD (Fig. 2(c)), and it was significantly suppressed by feeding HFLGD compared with feeding HFD. The percentage of $\mathrm{CD} 11 \mathrm{c}^{-} \mathrm{CD} 206^{+}$cells (M2 macrophages) significantly decreased by feeding HFD compared with feeding NFD, and it was increased by feeding HFLGD compared with feeding HFD (Fig. 2(d)). The M1:M2 macrophage ratio was significantly increased by feeding HFD compared with feeding NFD, and it was significantly suppressed in mice fed HFLGD compared with mice fed HFD (Fig. 2(e)).

\section{Real-time PCR analysis of pro-inflammatory gene expression in epididymal fat tissue}

Feeding HFD elevated the expression levels of macrophage marker genes (Ccr2 and Cd68) and pro-inflammatory genes (Ccl2, Il6, Nos2, Thf and Lep) compared with feeding NFD (Fig. 3). In mice fed HFLGD, the expressions of Ccr2 and Lep were significantly down-regulated compared with HFD. Although not significant, expression levels of the $\mathrm{Ccl} 2$ gene was down-regulated by feeding HFLGD $(P=0.06)$, and the other genes examined ( $C d 68$, Il6, Tnf and Nos2) tended to be downregulated in mice fed HFLGD.

\section{Serological analysis aimed to assess insulin resistance}

Mouse serum was collected at the end of the feeding period. Fasting blood glucose levels were slightly increased in the HFD-fed group compared with the NFD-fed group, although it was not significant (Fig. 4(a)). Feeding HFLGD significantly decreased fasting glucose levels compared with feeding HFD. Fasting blood insulin levels and the HOMA-IR index were significantly increased by HFD feeding compared with NFD feeding (Fig. 4(b) and (c)). HFLGD feeding did not suppress blood insulin levels and the HOMA-IR index compared with HFD feeding. The HOMA-IR index was significantly correlated with the M1:M2 macrophage ratio (Fig. 4(d)).

\section{Discussion}

In a diet-induced obese model, some LAB have been shown to improve obesity and insulin resistance that closely relate to chronic inflammation, to which macrophage infiltration largely contributes $^{(5-9)}$. The present study demonstrates that, on a cell basis by flow cytometry, LG2055 administration prevented infiltration of macrophages into the adipose tissue of mice fed an HFD, which had been suggested by a previous study ${ }^{(17)}$ showing that expression of the $\mathrm{Ccl} 2$ gene, a crucial factor for macrophage infiltration, was down-regulated in diet-induced obese mice. 

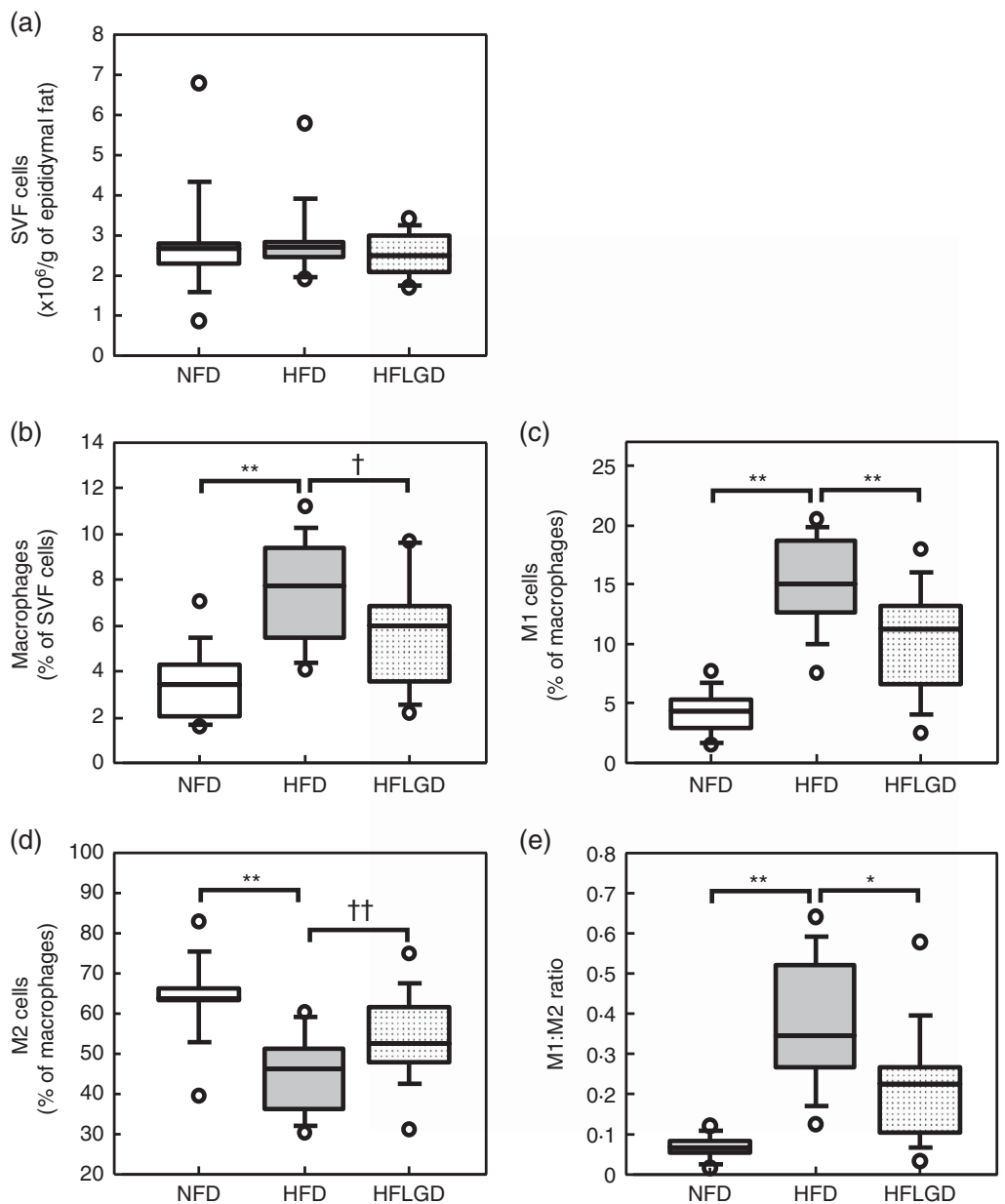

Fig. 2. Population analysis of macrophages in epididymal fat tissue. (a) Stromal vascular fraction (SVF) cells were counted using a Celltac haematology analyser and the number of cells in $1 \mathrm{~g}$ of epididymal fat tissue was calculated. Figures show the percentage of (b) macrophage population in live SVF cells; (c) M1 macrophage and (d) M2 macrophage population from all macrophages; (e) shows the ratio of M1:M2 macrophages. $\square$, Values of the normal-fat diet (NFD) fed group; $\square$, values of the high-fat diet (HFD) fed group; and $\because$, values of the HFD containing LG2055 (HFLGD) fed group. Data are shown as box plots ( $n$ 12); the centre line of each box represents the median; the top and bottom of the boxes represent the 25th and 75th percentiles of the data, respectively; and the top and the bottom of the error bars represent the 10th and 90th percentiles of the data, respectively. Statistically significant difference: ${ }^{*} P<0.05$, ${ }^{\star *} P<0.01$ (Dunnett's test). Borderline significant difference: $\dagger P=0.08$, †† $P=0.06$ (Dunnett's test).

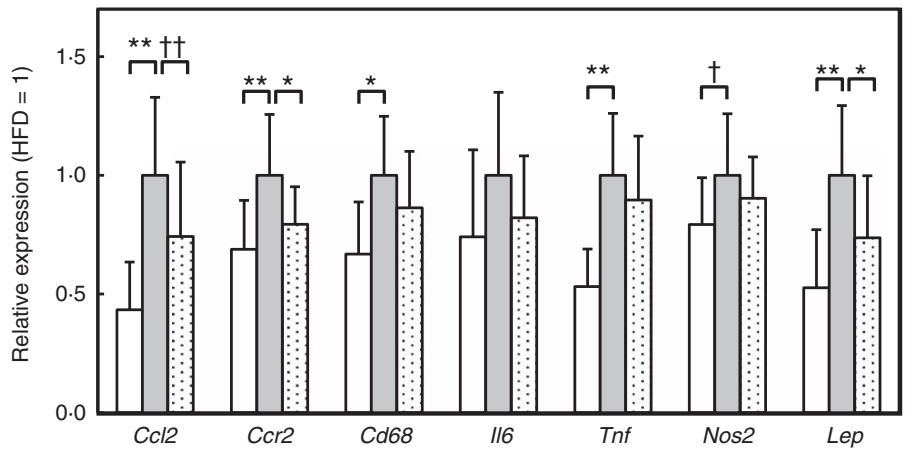

Fig. 3. Relative RNA expression in epididymal fat tissue. Gene expression levels in each diet group were determined by real-time PCR analysis. The expression levels of target genes were normalised with endogenous control genes, $\beta$-actin and glyceraldehyde-3-phosphate dehydrogenase, and the data were analysed according to the $2^{-\Delta \Delta c_{t}}$ method. $\square$, Values of the normal-fat diet group; $\square$, values of the high-fat diet (HFD) fed group; $\because$, HDF containing LG2055-fed group. The data are shown as relative values based on the expression levels in the HFD-fed group. Values are means ( $n$ 12), with standard deviations represented by vertical bars. Statistically significant difference: ${ }^{*} P<0.05,{ }^{*} P<0.01$ (Dunnett's test). Borderline significant difference: $† P=0.10$, †† $P=0.06$ (Dunnett's test).

A number of studies have reported that obesity promotes macrophage infiltration into adipose tissue ${ }^{(29)}$ : invading macrophages then polarise to pro-inflammatory M1 macrophages and exacerbate inflammation by pro-inflammatory cytokines produced there; the cytokines then activate the JNK and IKK $\beta$ signalling pathways, leading to exacerbation of insulin 
(a)

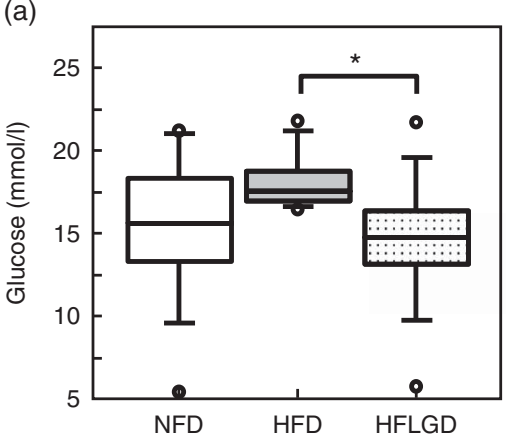

(b)

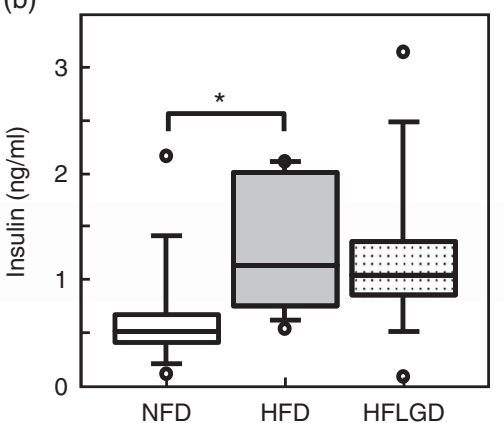

(c)

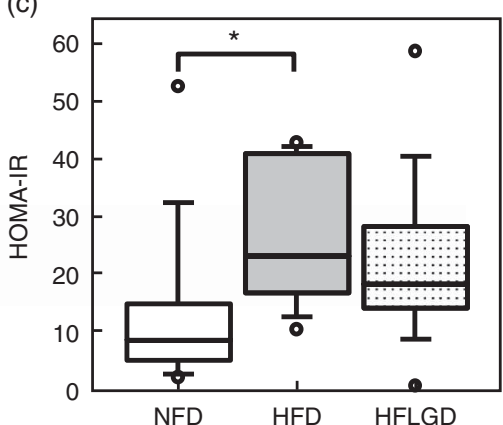

(d)

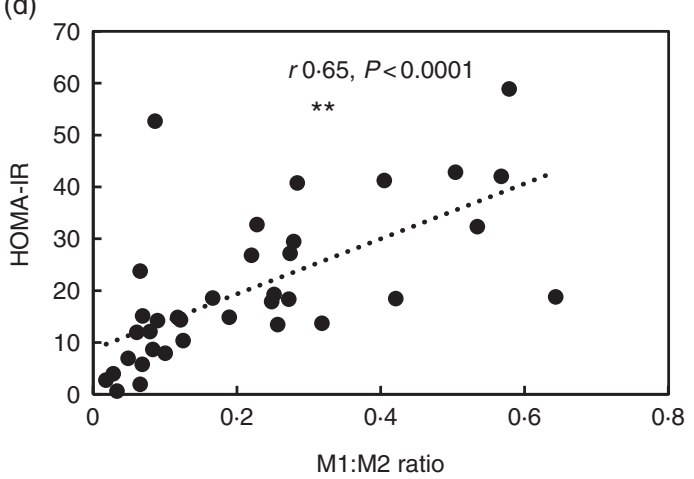

Fig. 4. Serological analysis of mouse serum. Mouse serum was collected after 12 weeks of feeding. Fasting glucose (a) and insulin (b) levels were analysed. The index of homoeostasis model assessment for insulin resistance (HOMA-IR) (c) was calculated using the following formula: fasting glucose (mmol/l) $\times$ fasting insulin $(\mathrm{mU} / \mathrm{ml}) / 22 \cdot 5$. $\square$, Values of the normal-fat diet (NFD) group; $\square$, values of the high-fat diet (HFD) fed group; F, values of the HDF containing LG2055 (HFLGD) fed group. Data are shown as box plots $(n 12)$, as described in Fig. 2. Correlation between the HOMA-IR index and the M1:M2 macrophage ratio was plotted (d) with Pearson's $r$ correlation and the corresponding $P$ value. Statistically significant difference: ${ }^{\star} P<0.05$ (Dunnett's test).

resistance $^{(30,31)}$. It is reported that an increase in the M1:M2 ratio is associated with an increase in systemic inflammation and induction of insulin resistance ${ }^{(19)}$. Chronic inflammation and insulin resistance are critical in exacerbating the symptoms of obesity-induced metabolic diseases such as CVD and type 2 diabetes ${ }^{(21,29)}$. Inhibition of macrophage infiltration into adipose tissue and down-regulation of pro-inflammatory M1 macrophages must contribute to amelioration of these diseases. It has been reported that a CCR2 antagonist, an inhibitor of macrophage infiltration, ameliorated obesity-associated inflammation and insulin resistance in mice ${ }^{(32)}$. Furthermore, positive effects of CCR2 antagonist have been recently shown in human diabetic patients ${ }^{(29,33)}$

In the present study, gene expression and flow cytometric analyses revealed that an increase in macrophage invasion into adipose tissue caused by feeding an HFD was suppressed by the additional feeding of LG2055 (Fig. 2 and 3). Flow cytometric analysis also revealed that the abundance in M1 macrophages and the M1:M2 macrophage ratio were significantly increased in the adipose tissue after feeding the HFD and significantly suppressed by the additional feeding of LG2055 (Fig. 2). Some studies on the abundance of M1 macrophages in the adipose tissue of diet-induced obese mice reported that approximately $25-70 \%$ of adipose tissue macrophages were M1 macrophages ${ }^{(19,26,34)}$. In our study, the mean percentage of M1 macrophages in the HFD-fed group was approximately $15 \%$, which could be low compared with other reports. Nevertheless, the suppressive effect of LG2055 on macrophage invasion was clearly demonstrated.

The present study minimised an influence of granulocytes contamination on the flow cytometric analysis of macrophages. Cho et $a l .{ }^{(26)}$ reported that the $\mathrm{F} 4 / 80{ }^{\mathrm{mid}} \mathrm{CD} 11 \mathrm{~b}{ }^{\mathrm{mid} / \mathrm{high}}$ population in SVF cells from adipose tissue contains neutrophils and eosinophils; therefore, only the $\mathrm{F} 4 / 80{ }^{\text {high }} \mathrm{CD} 11 \mathrm{~b}{ }^{\text {high }}$ population should be gated to minimise contamination. In our study, the $\mathrm{F} 4 / 80^{\text {high }} \mathrm{CD} 11 \mathrm{~b}{ }^{\text {high }}$ population was defined as macrophages according to the method of Cho et al. In addition, even if the $\mathrm{F} 4 / 80^{+} \mathrm{CD} 11 \mathrm{~b}^{+}$cells were defined as macrophages, the results of this study were not affected: the percentage of M1 macrophages and the M1:M2 ratio in the HFD-fed group were higher than those in the HFLGD-fed group (data not shown).

It is known that the gene expression levels of pro-inflammatory cytokines increase together with an increase in macrophage invasion in the adipose tissue of diet-induced obese mice ${ }^{(18,29)}$. In our study, the expression levels of pro-inflammatory cytokine genes such as Tnf and Lep were also increased by feeding a HFD, and the levels of lep were down-regulated by the additional feeding of LG2055 (Fig. 3). Leptin, the translational product of the Lep gene, known to be a hormone produced by adipocytes, which influences energy homoeostasis, is also reported to be a cytokine that induces pro-inflammatory cytokine secretion from macrophages and monocytes $^{(35)}$. Dib et al. ${ }^{(36)}$ showed that obesity-associated inflammation in adipose tissue was suppressed when the leptin 
receptor was absent on the cells derived from the bone marrow, suggesting the importance of leptin signal in the exacerbation of obesity-associated inflammation. Our results showing downregulation of Lep also suggested that inflammation in adipose tissue was ameliorated by the administration of LG2055.

Based on flow cytometric and gene expression analysis data, it was suggested that administration of LG2055 might improve insulin resistance, which is closely related to the status of inflammation in adipose tissue ${ }^{(19,29)}$. Our results show that fasting glucose was significantly reduced by the administration of LG2055, although the HOMA-IR index was not significantly decreased (Fig. 4). Interestingly, a significant correlation was observed between the HOMA-IR index and the M1:M2 macrophage ratio (Fig. $4(d)$ ), suggesting that the reduced M1: M2 ratio by feeding LG2055 might lead to the improvement of the insulin resistance.

Although much remains to be elucidated regarding how LG2055 suppresses macrophage invasion and adipose tissue inflammation, a compositional change in the gut microbiota could be a factor for the suppression. Wang et al. ${ }^{(37)}$ showed that the administration of probiotic Lactobacillus and Bifidobacterium modulated the gut microbiota and attenuated adipose tissue inflammation, macrophage invasion and insulin resistance in HFD-fed mice. A number of studies have demonstrated that the composition of the gut microbiota is altered in obese mice and humans as well as in type 2 diabetes patients. The compositional change in the gut microbiota is known to affect the amount of SCFA and secondary bile acids in the intestine, as well as lipopolysaccharide levels in the blood, which induce inflammatory immune responses and exacerbate inflammation $^{(38,39)}$. Although we have not assessed the effect of LG2055 on the gut microbiota, Sakai et al. ${ }^{(40)}$ reported that the administration of LG2055 increased the IgA antibody production in the intestine of mice. Given that the secreted IgA antibody has the potential to modify the composition of microbiota $^{(41)}$, the administration of LG2055 could affect the state of inflammation through the compositional alteration.

In this study, body weight and adipose tissue weight were not significantly decreased by feeding $45 \%$ energy fat $(24 \%$, w/w fat) diet plus LG2055 for 12 weeks (Table 1). In contrast, Miyoshi et $a l .{ }^{(17)}$ reported reduced body and adipose tissue weights after the consumption of LG2055 using a diet-induced obese mouse model, in which the diet used contained $10 \%(\mathrm{w} / \mathrm{w})$ fat and the feeding period was 24 weeks. Thus, the difference in fat content in the diet and the feeding period might produce the discrepancy in the body and adipose tissue weight results between the studies. The present study used a $45 \%$ energy fat-containing diet, because Thomas et al. ${ }^{(42)}$ demonstrated that the intake of a $45 \%$ energy fat diet significantly induced macrophage invasion and adipose tissue inflammation in mice, which were not significantly induced by the feeding of $10 \%(\mathrm{w} / \mathrm{w})$ fat diet according to Miyoshi et $a l^{(17)}$

In summary, administration of LG2055 to mice fed an HFD significantly suppressed infiltration of pro-inflammatory M1 macrophages, chemokine receptor gene ( $C c r 2)$ expression, which is responsible for macrophage infiltration and adipokine gene (Lep) expression, which is capable of inducing proinflammatory cytokines from macrophages, in adipose tissue.
A significant reduction in fasting glucose levels in the blood was also observed. Taken together, LG2055 ingestion is suggested to bring about beneficial properties such as anti-inflammatory tendencies and a possible involvement in improving insulin resistance.

\section{Acknowledgements}

All authors are employed by Megmilk Snow Brand Co. Ltd. The authors are grateful to Ken Kato, Yuko Ishida, Akihiro Ogawa, Maya Yamashita, Michio Kawano, Kurumi Takagi, Hiroshi Uenishi and Satoshi Higurashi (Megmilk Snow Brand Co. Ltd) for their skilful collaboration and useful suggestions. The authors also appreciate the work of Emiko Yamaguchi (YBS Co. Ltd) in preparing the experimental diets and technical assistance, and Masaki Kumai, Shigeki Kanoh and Mie Kasuga (YBS Co. Ltd) for animal care.

This research received no specific grant from any funding agency, commercial or not-for-profit sectors.

K. U. designed the study, carried out experimental work, analysed data and wrote the manuscript. M. M. designed the study and carried out experimental work. Y. K. designed the study.

The authors declare no conflicts of interest.

\section{References}

1. Hammes WP \& Tichaczek PS (1994) The potential of lactic acid bacteria for the production of safe and wholesome food. Z Lebensm Unters Forsch 198, 193-201.

2. Hill C, Guarner F, Reid G, et al. (2014) Expert consensus document. The International Scientific Association for Probiotics and Prebiotics consensus statement on the scope and appropriate use of the term probiotic. Nat Rev Gastroenterol Hepatol 11, 506-514.

3. Tsai YT, Cheng PC \& Pan TM (2012) The immunomodulatory effects of lactic acid bacteria for improving immune functions and benefits. Appl Microbiol Biotechnol 96, 853-862.

4. Jan RL, Yeh KC, Hsieh MH, et al. (2012) Lactobacillus gasseri suppresses Th17 pro-inflammatory response and attenuates allergen-induced airway inflammation in a mouse model of allergic asthma. Br J Nutr 108, 130-139.

5. Lee HY, Park JH, Seok SH, et al. (2006) Human originated bacteria, Lactobacillus rhamnosus LP60, produce conjugated linoleic acid and show anti-obesity effects in diet-induced obese mice. Biochim Biophys Acta 1761, 736-744.

6. Zhao X, Higashikawa F, Noda M, et al. (2012) The obesity and fatty liver are reduced by plant-derived Pediococcus pentosaceus LP28 in high fat-diet induced obese mice. PLOS ONE 7, e30696.

7. Park JE, Oh SH \& Cha YS (2013) Lactobacillus plantarum LG42 isolated from gajami sik-hae decreases body and fat pad weights in diet-induced obese mice. J Appl Microbiol 116, 145-156.

8. Park DY, Ahn YT, Park SH, et al. (2013) Supplementation of Lactobacillus curvatus HY7601 and Lactobacillus plantarum KY1032 in diet-induced obese mice is associated with gut microbial changes and reduction in obesity. PLOS ONE 8, e59470.

9. Tsai YT, Cheng PC \& Pan TM (2014) Anti-obesity effects of gut microbiota are associated with lactic acid bacteria. Appl Microbiol Biotechnol 98, 1-10. 
10. Fujiwara Y, Seto A \& Hashiba H (2001) Establishment of orally-administered Lactobacillus gasseri SBT2055SR in the gastrointestinal tract of humans and its influence on intestinal microflora and metabolism. J Appl Microbiol 90, 343-352.

11. Nakayama Y, Moriya T, Sakai F, et al. (2014) Oral administration of Lactobacillus gasseri SBT2055 is effective for preventing influenza in mice. Sci Rep $\mathbf{4}, 4638$.

12. Sato M, Uzu K, Yoshida T, et al. (2008) Effects of milk fermented by Lactobacillus gasseri SBT2055 on adipocyte size in rats. Br J Nutr 99, 1013-1017.

13. Hamad EM, Sato M, Uzu K, et al. (2009) Milk fermented by Lactobacillus gasseri SBT2055 influences adipocyte size via inhibition of dietary fat absorption in Zucker rats. Br J Nutr 101, 716-724.

14. Kadooka Y, Ogawa A, Ikuyama K, et al. (2011) The probiotic Lactobacillus gasseri SBT2055 inhibits enlargement of visceral adipocytes and up-regulation of serum soluble adhesion molecule (sICAM-1) in rats. Int Dairy J 21, 623-627.

15. Kadooka Y, Sato M, Imaizumi K, et al. (2010) Regulation of abdominal adiposity by probiotics (Lactobacillus gasseri SBT2055) in adults with obese tendencies in a randomized controlled trial. Eur J Clin Nutr 64, 636-643.

16. Kadooka Y, Sato M, Ogawa A, et al. (2013) Effect of Lactobacillus gasseri SBT2055 in fermented milk on abdominal adiposity in adults in a randomised controlled trial. Br J Nutr 110, 1696-1703.

17. Miyoshi M, Ogawa A, Higurashi S, et al. (2014) Anti-obesity effect of Lactobacillus gasseri SBT2055 accompanied by inhibition of pro-inflammatory gene expression in the visceral adipose tissue in diet-induced obese mice. Eur J Nutr 53, 599-606.

18. Chawla A, Nguyen KD \& Goh YP (2011) Macrophagemediated inflammation in metabolic disease. Nat Rev Immunol 11, 738-749.

19. Fujisaka S, Usui I, Bukhari A, et al. (2009) Regulatory mechanisms for adipose tissue M1 and M2 macrophages in diet-induced obese mice. Diabetes 58, 2574-2582.

20. Xu H, Barnes GT, Yang Q, et al. (2003) Chronic inflammation in fat plays a crucial role in the development of obesity-related insulin resistance. J Clin Invest 112, 1821-1830.

21. Lumeng CN \& Saltiel AR (2011) Inflammatory links between obesity and metabolic disease. J Clin Invest 121, 2111-2117.

22. Kamei N, Tobe K, Suzuki R, et al. (2006) Overexpression of monocyte chemoattractant protein-1 in adipose tissues causes macrophage recruitment and insulin resistance. $\mathrm{J}$ Biol Chem 281, 26602-26614.

23. Kanda H, Tateya S, Tamori Y, et al. (2006) MCP-1 contributes to macrophage infiltration into adipose tissue, insulin resistance, and hepatic steatosis in obesity. J Clin Invest 116, 1494-1505.

24. Weisberg SP, Hunter D, Huber R, et al. (2006) CCR2 modulates inflammatory and metabolic effects of high-fat feeding. J Clin Invest 116, 115-124.

25. Nishimura S, Manabe I, Nagasaki M, et al. (2009) CD8+ effector $\mathrm{T}$ cells contribute to macrophage recruitment and adipose tissue inflammation in obesity. Nat Med 15, 914-920.
26. Cho KW, Morris DL \& Lumeng CN (2014) Flow cytometry analyses of adipose tissue macrophages. Methods Enzymol 537, 297-314.

27. Vandesompele J, De Preter K, Pattyn F, et al. (2002) Accurate normalization of real-time quantitative RT-PCR data by geometric averaging of multiple internal control genes. Genome Biol 3, RESEARCH 0034.

28. Matthews DR, Hosker JP, Rudenski AS, et al. (1985) Homeostasis model assessment: insulin resistance and $\beta$-cell function from fasting plasma glucose and insulin concentrations in man. Diabetologia 28, 412-419.

29. McNelis JC \& Olefsky JM (2014) Macrophage, immunity, and metabolic disease. Immunity $\mathbf{4 1}, 36-48$.

30. Solinas G \& Karin M (2010) JNK1 and IKK $\beta$ : molecular links between obesity and metbabolic dysfunction. FASEB J $\mathbf{2 4}$, $2596-2611$

31. Gregor MF \& Hotamisligil GS (2011) Inflammatory mechanisms in obesity. Annu Rev Immunol 29, 415-445.

32. Kang YS, Lee MH, Song HK, et al. (2010) CCR2 antagonism improves insulin resistance, lipid metabolism, and diabetic nephropathy in type 2 diabetic mice. Kidney Int $\mathbf{7 8}$, 883-894.

33. Di Prospero NA, Artis E, Andrade-Gordon P, et al. (2014) CCR2 antagonism in patients with type 2 diabetes mellitus: a randomized, placebo-controlled study. Diabetes Obes Metab 16, 1055-1064.

34. Ying W, Kanameni S, Chang CA, et al. (2014) Interferon tau alleviates obesity-induced adipose tissue inflammation and insulin resistance by regulating macrophage polarization. PLOS ONE 9, e98835.

35. Carbone F, La Rocca C \& Matarese G (2012) Immunological functions of leptin and adiponectin. Biochimie $\mathbf{9 4}$, 2082-2088.

36. Dib LH, Ortega MT, Fleming SD, et al. (2014) Bone marrow leptin signalling mediates obesity-associated adipose tissue inflammation in male mice. Endocrinology 155, 40-46.

37. Wang J, Tang H, Zhang C, et al. (2015) Modulation of gut microbiota during probiotic-mediated attenuation of metabolic syndrome in high fat diet-fed mice. ISME J 9, 1-15.

38. Musso G, Gambino R \& Cassader M (2011) Interactions between gut microbiota and host metabolism predisposing to obesity and diabetes. Annu Rev Med 62, 361-380.

39. Belkaid Y \& Hand TW (2014) Role of the microbiota in immunity and inflammation. Cell 157, 121-141.

40. Sakai F, Hosoya T, Ono-Oumachi A, et al. (2014) Lactobacillus gasseri SBT2055 induces TGF- $\beta$ expression in dendritic cells and activates TLR2 signal to produce IgA in the small intestine. PLOS ONE 9, e105370.

41. Kawamoto S, Tran TH, Maruya M, et al. (2012) The inhibitory receptor PD-1 regulates IgA selection and bacterial composition in the gut. Science 336, 485-489.

42. Thomas AP, Dunn TN, Oort PJ, et al. (2011) Inflammatory phenotyping identifies CD11d as a gene markedly induced in white adipose tissue in obese rodents and women. J Nutr 141, $1172-1180$ 